\title{
Longitudinal changes in the height and location of bone bridge from autogenous iliac bone graft in patients with cleft lip and palate
}

\author{
Kotaro Tanimoto ${ }^{1 *}$, Yuki Tanne ${ }^{1}$, Keisuke Sumi ${ }^{1}$, Naoto Hirose ${ }^{1}$, Nobuhiko Kawai ${ }^{2}$, Eiji Tanaka ${ }^{2}$, \\ Kazuo Tanne ${ }^{1}$ \\ ${ }^{1}$ Department of Orthodontics, Applied Life Sciences, Hiroshima University Institute of Biomedical \& Health Sciences, Hiroshima, \\ Japan \\ ${ }^{2}$ University of Tokushima Graduate School of Oral Sciences, Tokushima, Japan \\ Email: *tkotaro@hiroshima-u.ac.jp
}

Received 20 January 2013; revised 1 March 2013; accepted 9 March 2013

\begin{abstract}
Background: Secondary alveolar bone graft has been widely used for the closure of maxillary bone defects in patients with cleft lip and palate (CLP). However, the log-term stability of grafted bone in detail remains unclear. Purpose: To evaluate the nature of longitudinal changes in the grafted bone from autogenous iliac bone in patients with CLP for more than 2 years after the surgery. Methods and Subjects: The subjects were 124 CLP patients treated with iliac bone graft in Hiroshima University Hospital from 1997 to 2007. The height and location of the bone bridge was evaluated radiographically before and after surgery. Results: 1) Bone graft was performed at a mean age of $12.5( \pm 4.2)$ years old. The canines on the cleft side were not erupted at the surgery in $60.0 \%$. All canines erupted except for the congenital missing and impacted ones $(\mathbf{1 1 . 3 \% )}$ ) within 2 years after bone graft; 2) The height of the bone bridge was more than $11 \mathrm{~mm}$ in $\mathbf{7 1 . 8 \%}$ of all patients 1 month after surgery. In addition, the location of bone bridge in $66.1 \%$ of all patients were clinically satisfactory. However, these rates decreased time-dependently owing to bone resorption after the bone graft. Conclusions: In conclusion, it is shown that autogenous bone graft produce a sufficient bone bridge in the jaw cleft area by the long-term evaluation of the changes in height and location of bone bridge, although some amount of time-dependent bone resorption is inevitable.
\end{abstract}

Keywords: Bone Bridge; Bone Graft; Canine Eruption; Cleft Lip and Palate

\section{INTRODUCTION}

Cleft lip and palate (CLP) is a prevalent congenital ano-

"Corresponding author. maly in the orofacial region characterized by a jaw cleft due to failure of the palatal shelves to fuse properly. CLP is caused by various genetic and environmental factors, and the incidence rate of CLP is $0.19 \%$ in Japan [1]. Generally, CLP patients receive labioplasty and initial palatoplasty at the infant stage as the initial treatment, and speech therapy is also needed for the recovery of speech function. In addition, discontinuity of the upper dental arch due to the jaw cleft frequently causes malocclusion, and orthodontic treatment is conducted on most CLP patients for acquisition of stable occlusion.

Boyne and Sands [2] first reported the benefit of secondary alveolar bone graft for patients with CLP. Since then, various studies have been conducted and indicated excellent results to support the availability of bone graft [3-11]. This procedure makes it possible to induce eruption of the canine to newly formed alveolar bone and to develop maxillary dental arch and occlusion without prosthetic treatment. Therefore, alveolar bone graft is essential for the achievement of optimal stomatognathic morphology and function. It is also of a great importance to assess the outcome of bone graft and to clarify key determinants for successful results. From an orthodontic point of view, the most important advantage of bone graft is to complete the upper dentition with continuous alveolar bone and erupted canines at the cleft site, whereas only undesirable results can be achieved by insufficient formation of the bone bridge.

Various factors to affect the outcome of secondary bone graft were discussed in previous studies [5,7,12-15]. Dental development age at the time of surgery is important for the success of bone graft [5,12-15]. It is recommended to perform bone graft during mixed dentition before the full eruption of canines to allow canine eruption into the grafted area [5,12-15]. Another factor, type of CLP, might affect the outcome of bone graft, that is, the success rate is less in bilateral CLP (BCLP) than in 
the unilateral one (UCLP) [15].

The purpose of this study was to evaluate the nature of longitudinal changes in bone graft into alveolar clefts using cancellous iliac bone in CLP patients for a period of 2 years after the surgery.

\section{MATERIALS AND METHODS}

The subjects were 124 CLP patients treated with iliac bone graft in Hiroshima University Hospital from 1992 to 2007. Gender, cleft type, age, eruption of canines, and history of orthodontic treatment were examined. For the history of orthodontic treatment, stage of orthodontic treatment at bone graft was evaluated.

Radiographic analysis was carried out by means of periapical and panoramic X-ray films before and after surgery for bone graft (T0: immediately before surgery, T1: 1 month after surgery, T2: 1 year after surgery, T3: 2 years after surgery). The height of the bone bridge was determined on X-ray films and classified into 4 grades (Figure 1); Grade 0 = bone bridge is undetectable, Grade 1 = vertical height of bone bridge is 0 to until $5 \mathrm{~mm}$, Grade 2 = vertical height of bone bridge is 5 to $11 \mathrm{~mm}$, Grade 3 = vertical height of bone bridge is more than 11 $\mathrm{mm}$ or complete bridging.

The location of bone bridge was determined on X-ray films and classified into6categories according to the Chelsea alveolar bone graft scale reported by Witherow et al. [9]. The bone bridge located up to the amelocemental junction, and covers more than $75 \%$ and $25 \%$ $75 \%$ of the root surface was classified into Category A and Category B, respectively. The bone bridge with less than $25 \%$ and $50 \%$ uncovered root surface from the amelocemental junction was classified into Category C and Category D, respectively. Category E does not have the bone bridge at either the apical or the amelocemental levels. The bone bridge of Category $\mathrm{F}$ has $75 \%$ or greater uncovered root surface from the amelocemental junction.

For the evaluation of canine eruption, patients with congenital missing (7 patients) or abnormally unerupted (7 patients) canine on the cleft side were excluded. The degree of canine eruption on the cleft side was classified into3 scores by means of a panoramic X-ray: Score $1=$ not erupted from alveolar bone and tooth root formation is not completed, Score 2 = erupted and tooth root is incomplete in the formation, Score $3=$ erupted and tooth root formation is completed.

Postoperative changes in the height and location of the bone bridge and the degree of canine eruption were investigated.

\section{RESULTS}

The number of BCLP patients was 32 (25.8\%) and that of UCLP patients was 92 (74.2\%) (Table 1). Bone graft

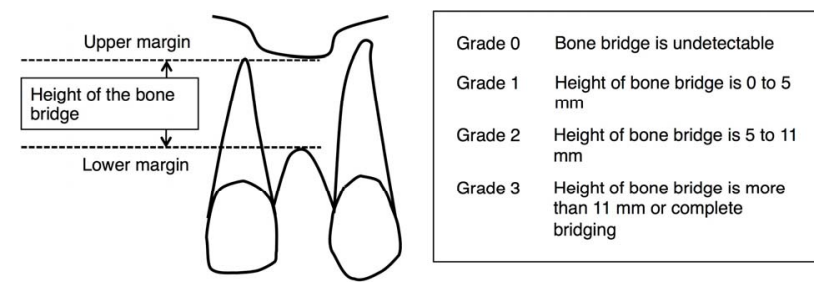

Figure 1. Evaluation of the height of the bone bridge. The height of the bone bridge was determined on periapical X-ray films and classified into 4 grades (Grade 0 -Grade 3 ).

Table 1. Summary of patients $(\mathrm{N}=124)$.

\begin{tabular}{ccccc}
\hline & \multirow{2}{*}{ BCLP } & \multicolumn{2}{c}{ UCLP } & \multirow{2}{*}{ Total } \\
\cline { 3 - 4 } & & Right & Left & \\
\hline Male & $26(21.0 \%)$ & $14(11.3 \%)$ & $48(38.7 \%)$ & $88(71.0 \%)$ \\
Female & $6(4.8 \%)$ & $14(11.3 \%)$ & $16(12.9 \%)$ & $36(29.0 \%)$ \\
Total & $32(25.8 \%)$ & $28(22.6 \%)$ & $64(51.6 \%)$ & $124(100 \%)$ \\
\hline
\end{tabular}

was performed at a mean age of $12.5( \pm 4.2)$ years old. The surgery was carried out at the observation period after the first phase orthodontic treatment in 75 (60.5\%) patients and during the first phase treatment in 27 (21.8\%) patients (Table 2). The percentage of unerupted canineson the cleft side (Score 1 ) was $60.0 \%$ at T0, and all canines were erupted two years after the bone graft (Table 3).

The bone bridge at the bone graft area with more than $11 \mathrm{~mm}$ height (Grade 3 ) was shown in $71.8 \%$ of patients at T1, and the rate decreased at T2 and T3 in stages (Table 4). The rate of patients in Grade 2 and Grade 1 obviously increased from $21.0 \%$ to $37.1 \%$ and $11.3 \%$ to $17.7 \%$ from T2 to T3, respectively. The bone bridge was not shown (Grade 0 ) in $4.0 \%$ of patients at $\mathrm{T} 1$, and the rate increased to $8.1 \%$ at $\mathrm{T} 3$.

The bone bridge located up to the amelocemental junction, with enough coverage of the root surface (Category A) was shown in $66.1 \%$ of patients at $\mathrm{T} 1$, and the rate decreased to $60.5 \%$ and $37.1 \%$ at $\mathrm{T} 2$ and $\mathrm{T} 3$, respectively (Table 5). The rate of patients in Category B, the bone bridge with less root surface coverage than Category $\mathrm{A}$, was $4.8 \%, 6.5 \%$, and $5.6 \%$ at $\mathrm{T} 1, \mathrm{~T} 2$, and $\mathrm{T} 3$, respectively. The rate of patients in Category $\mathrm{B}, \mathrm{C}, \mathrm{D}$, and $\mathrm{F}$ increased at $\mathrm{T} 2$ and $\mathrm{T} 3$, suggesting the resorption of the coronal side of grafted bone.

\section{DISCUSSION}

Since the introduction, secondary alveolar bone graft has been successfully used for the improvement of stomatognathic morphology and function in CLP patients.

In this study, bone graft was performed at an age of $12.5( \pm 4.2)$ years old on average and $62.1 \%$ of the upper canines on the cleft side were not erupted at the time of surgery. Timing of the surgery seems a little late in 
Table 2. Stage of orthodontic treatment at bone graft.

\begin{tabular}{cc}
\hline Stage & Number of patients \\
\hline Before orthodontic treatment & $4(3.2 \%)$ \\
During 1st phase treatment & $27(21.8 \%)$ \\
Follow-up period (after 1st phase) & $75(60.5 \%)$ \\
During 2nd phase treatment & $16(12.9 \%)$ \\
Retention period (after 2nd phase) & $2(1.6 \%)$ \\
\hline
\end{tabular}

Table 3. Longitudinal changes in canine eruption on the cleft side.

\begin{tabular}{cccc}
\hline Step of the treatment & Score 1 & Score 2 & Score 3 \\
\hline T0 & $66(60.0 \%)$ & $18(16.4 \%)$ & $26(23.6 \%)$ \\
T1 & $27(24.5 \%)$ & $50(45.5 \%)$ & $33(30.0 \%)$ \\
T2 & $15(13.6 \%)$ & $47(42.7 \%)$ & $48(43.6 \%)$ \\
T3 & $0(0 \%)$ & $43(39.1 \%)$ & $67(60.9 \%)$ \\
\hline
\end{tabular}

Score $1=$ not erupted from alveolar bone and tooth root is incomplete; Score 2 = erupted and tooth root is incomplete; Score 3 = erupted and tooth root is complete; Patients with congenital missing or abnormally unerupted canine on the cleft side were excluded $(n=110)$.

Table 4. Longitudinal changes in the height of bone bridge.

\begin{tabular}{ccccc}
\hline $\begin{array}{c}\text { Step of the } \\
\text { treatment }\end{array}$ & Grade 0 & Grade 1 & Grade 2 & Grade 3 \\
\hline T0 & $112(90.3 \%)$ & $12(9.7 \%)$ & $0(0 \%)$ & $0(0 \%)$ \\
T1 & $5(4.0 \%)$ & $6(4.8 \%)$ & $24(19.4 \%)$ & $89(71.8 \%)$ \\
T2 & $6(4.8 \%)$ & $14(11.3 \%)$ & $26(21.0 \%)$ & $78(62.9 \%)$ \\
T3 & $10(8.1 \%)$ & $22(17.7 \%)$ & $46(37.1 \%)$ & $46(37.1 \%)$ \\
\hline
\end{tabular}

Table 5. Longitudinal changes in the location of bone bridge.

\begin{tabular}{ccccccc}
\hline $\begin{array}{c}\text { Step of the } \\
\text { treatment }\end{array}$ & $\mathrm{A}$ & $\mathrm{B}$ & $\mathrm{C}$ & $\mathrm{D}$ & $\mathrm{E}$ & $\mathrm{F}$ \\
\hline \multirow{2}{*}{$\mathrm{T} 0$} & 0 & 0 & 0 & 0 & 0 & 124 \\
& $(0 \%)$ & $(\%)$ & $(\%)$ & $(0 \%)$ & $(0 \%)$ & $(100 \%)$ \\
$\mathrm{T} 1$ & 82 & 6 & 25 & 1 & 5 & 5 \\
& $(66.1 \%)$ & $(4.8 \%)$ & $(20.2 \%)$ & $(0.8 \%)$ & $(4.0 \%)$ & $(4.0 \%)$ \\
$\mathrm{T} 2$ & 75 & 8 & 21 & 9 & 5 & 6 \\
& $(60.5 \%)$ & $(6.5 \%)$ & $(16.9 \%)$ & $(7.3 \%)$ & $(4.0 \%)$ & $(4.8 \%)$ \\
$\mathrm{T} 3$ & 46 & 7 & 39 & 15 & 7 & 10 \\
& $(37.1 \%)$ & $(5.6 \%)$ & $(31.5 \%)$ & $(12.1 \%)$ & $(5.6 \%)$ & $(8.1 \%)$ \\
\hline
\end{tabular}

several cases but is regarded as acceptable according to a previous finding such that the optimal timing is during mixed dentition before the upper canines erupt, usually at 8 - 12 years [5,12-15]. CLP patients, in general, have a significantly higher risk of canine impaction than noncleft patients [16]. An important significance of secondary bone graft is to complete the maxillary dentition with continuous alveolar bone, achieved by the newly grafted bone, and to induce spontaneous migration of the adjacent canines into the bone graft area [17]. In the present study, although 7 patients (5.7\%) have canine im- paction, $86.4 \%$ and $100 \%$ of canines in the other patients were erupted 1 and 2 years after surgery, respectively. It is thus confirmed that the present outcome of bone graft surgery satisfactory for the promotion of canine eruption and dental rehabilitation.

In addition, this long-term follow-up study revealed the nature of postoperative changes in the height and location of the bone bridge. In previous studies, the success rate of bone graft has been reported as $86.0 \%$ : Trindade et al. [18]; 86.7\%: Collins et al. [15]; 96\%: Bergland et al. [3]; Witherow et al. [9]; 78\%.

In the present study, alveolar bone bridge with more than $11 \mathrm{~mm}$ height (Grade 3) was $71.8 \% 1$ month after surgery. By use of the Chelsea Scale for the evaluation of bone bridge location, most of the cases in Grade 3 was classified into Category A, and several cases were classified into Category $\mathrm{C}$ because of the deficiency of root surface coverage at the amelocemental junction. On the other hand, no cases with bone bridge height in Grade 2 were classified into Category A. Most cases in Grade 2 were classified into Category B or C, and rarely judged as Category D. In addition, no cases in Grade 1 were classified into Category A, B, or C.

Although Category A, B, and C were suggested to be clinically acceptable, long-term stability of Category B was unknown because of the lack of bone bridge at the apical side [9]. In the present study, most of the bone bridges in Category B were stable, and 1 Category B case changed to Category E 2 yeas after bone graft. In addition, the height of the bone bridge recovered by bone graft was reduced time-dependently after the surgery in many cases. In these patients, the location of bone bridge were changed frequently from Category $\mathrm{A}$ to $\mathrm{C}$ or from Category $\mathrm{C}$ to $\mathrm{D}$ accompanied by the reduction of bone bridge height, suggesting the decrease in grafted bone at the coronal side. The bone bridge was not shown in 4.0\% of subjects as 1 month after the bone graft, and the rate was increased to $8.1 \% 2$ years later. There are many factors that affect the long-term stability of grafted bone. One of the possible factors is such orthodontic treatment prior to the bone graft as lateral expansion of the maxillary dentition and the correction of anterior cross-bite. Application of maxillary lateral expansion is mainly needed to correct collapsed maxillary segments and to provide better access for a maxillofacial surgeon to perform bone graft. In addition, expansion of the upper dentition prior to the bone graft facilitates closure of the nasal mucosa by widening the cleft as well as improving the narrowed maxillary dentition [19]. Indeed, most CLP patients need alveolar expansion because of narrow alveolar arch. However, the effect of alveolar expansion on the outcome of bone grafting is still under discussion [14].

The autogenous iliac bone grafting has been frequently 
employed for the closure of bone defects at jaw cleft site, and reported to allow stabilization of the maxillary dental arch and periodontal tissue, eruption of canine into the grafted area, promotion of oronasal fistula closure, improvement of nasal symmetry, and orthodontic tooth movement and placement of dental implants into the cleft area [3-11]. However, the iliac bone graft, particularly classic large-incision, open techniques for bone graft harvesting from anterior and posterior parts of the iliac crest, is surgically invasive, leading to a large encumbrance for patients $[20,21]$. The common donor site complications for the anterior ilium are pain, sensory loss, gait disturbance, infection, and hematoma formation [22].

It is important to assess the long-term stability of bone graft and to elucidate the key determinants for successful outcome of the surgery. Therefore, more extensive investigation is highly required to evaluate the prognosis of bone graft surgery, leading to an establishment of optimal therapeutic system for CLP patients.

In conclusion, it is shown that autogenous bone graft produce a sufficient bone bridge in the jaw cleft area by the long-term evaluation of the changes in height and location of bone bridge, although some amount of bone resoption is inevitable. Thus, the bone graft is an effective method for recovery of maxillary bone defect due to CLP, leading to a generation of continuous alveolar bone and optimal occlusion with a definite canine eruption. In addition, it is highly anticipated to further improve the treatment procedure for CLP in terms of the long-term stability of regenerated bone.

\section{REFERENCES}

[1] Natsume, N., Suzuki, T. and Kawai, T. (1988) The prevalence of cleft lip and palate in Japanese. British Journal of Oral Maxillofacial Surgery, 26, 232-236. doi:10.1016/0266-4356(88)90168-4

[2] Boyne, P.J. and Sands, N.R. (1972) Secondary bone grafting of residual alveolar and palatal clefts. Journal of Oral Surgery, 30, 87-92.

[3] Bergland, O., Semb, G. and Abyholm, F.E. (1986) Elimination of the residual alveolar cleft by secondary bone grafting and subsequent orthodontic treatment. Cleft Palate Journal, 23, 175-205.

[4] Abyholm, F.E., Bergland, O. and Semb, G. (1981) Secondary bone grafting of alveolar clefts. A surgical/orthodontic treatment enabling a non-prosthodontic rehabilitation in cleft lip and palate patients. Scandinavian Journal of Plastic and Reconstructive Surgery and Hand Surgery, 15, 127-140. doi:10.3109/02844318109103425

[5] Sindet-Pedersen, S. and Enemark, H. (1985) Comparative study of secondary and late secondary bone-grafting in patients with residual cleft defects. Short-term evaluation. International Journal of Oral Surgery, 14, 389-398. doi:10.1016/S0300-9785(85)80071-5
[6] Amanat, N. and Langdon, J.D. (1991) Secondary alveolar bone grafting in clefts of the lip and palate. Journal of Cranio-Maxillofacial Surgery, 19, 7-14. doi:10.1016/S1010-5182(05)80265-3

[7] Kortebein, M.J., Nelson, C.L. and Sadove, A.M. (1991) Retrospective analysis of 135 secondary alveolar cleft grafts using iliac or calvarial bone. Journal of Oral and Maxillofacial Surgery, 49, 493-498. doi:10.1016/0278-2391(91)90172-I

[8] Long Jr., R.E., Spangler, B.E. and Yow, M. (1995) Cleft width and secondary alveolar bone graft success. The Cleft Palate-Craniofacial Journal, 32, 420-427. doi:10.1597/1545-1569(1995)032<0420:CWASAB >2.3.C $\underline{\mathrm{O} ; 2}$

[9] Witherow, H., Cox, S., Jones, E., Carr, R. and Waterhouse, N. (2002) A new scale to assess radiographic success of secondary alveolar bone grafts. The Cleft PalateCraniofacial Journal, 39, 255-260. doi:10.1597/1545-1569(2002)039<0255:ANSTAR>2.0.C $\mathrm{O} ; 2$

[10] El Deeb, M., Messer, L.B., Lehnert, M.W., Hebda, T.W. and Waite, D.E. (1982) Canine eruption into grafted bone in maxillary alveolar cleft defects. Cleft Palate Journal, 19, 9-16.

[11] Kindelan, J.D., Nashed, R.R. and Bromige, M.R. (1997) Radiographic assessment of secondary autogenous alveolar bone grafting in cleft lip and palate patients. The Cleft Palate-Craniofacial Journal, 34, 195-198. doi:10.1597/1545-1569(1997)034<0195:RAOSAA>2.3. $\underline{\mathrm{CO} ; 2}$

[12] Enemark, H., Sindet-Pedersen, S. and Bundgaard, M. (1987) Long-term results after secondary bone grafting of alveolar clefts. Journal of Oral and Maxillofacial Surgery, 45, 913-919. doi:10.1016/0278-2391(87)90439-3

[13] Jia, Y.L., James, D.R. and Mars, M. (1998) Bilateral alveolar bone grafting: A report of 55 consecutively-treated patients. European Journal of Orthodontics, 20, 299-307. doi:10.1093/ejo/20.3.299

[14] Newlands, L.C. (2000) Secondary alveolar bone grafting in cleft lip and palate patients. British Journal of Oral Maxillofacial Surgery, 38, 488-491. doi:10.1054/bjom.2000.0300

[15] Collins, M., James, D.R. and Mars, M. (1998) Alveolar bone grafting: A review of 115 patients. European Journal of Orthodontics, 20, 115-120. doi:10.1093/ejo/20.2.115

[16] Russell, K.A. and McLeod, C.E. (2008) Canine eruption in patients with complete cleft lip and palate. The Cleft Palate-Craniofacial Journal, 45, 73-80. doi:10.1597/07-049.1

[17] Da Silva Filho, O.G., Teles, S.G., Ozawa, T.O. and Filho, L.C. (2000) Secondary bone graft and eruption of the permanent canine in patients with alveolar clefts: Literature review and case report. Angle Orthodontist, 70, 174178.

[18] Trindade, I.K., Mazzottini, R., Silva Filho, O.G., Trindade, I.E. and Deboni, M.C. (2005) Long-term radiographic assessment of secondary alveolar bone grafting outcomes in patients with alveolar clefts. Oral Surgery, Oral 
Medicine, Oral Pathology, Oral Radiology, and Endodontology, 100, 271-277.

doi:10.1016/j.tripleo.2005.03.012

[19] Hall, H.D. and Posnick, J.C. (1983) Early results of secondary bone grafts in 106 alveolar clefts. Journal of Oral and Maxillofacial Surgery, 41, 289-294. doi:10.1016/0278-2391(83)90295-1

[20] Gimbel, M., Ashley, R.K., Sisodia, M., Gabbay, J.S., Wasson, K.L., Heller, J., Wilson, L., Kawamoto, H.K. and Bradley, J.P. (2007) Repair of alveolar cleft defects: Reduced morbidity with bone marrow stem cells in a re- sorbable matrix. Journal of Craniofacial Surgery, 18, 895-901. doi:10.1097/scs.0b013e3180a771af

[21] Heary, R.F., Schlenk, R.P., Sacchieri, T.A., Barone, D. and Brotea, C. (2002) Persistent iliac crest donor site pain: Independent outcome assessment. Neurosurgery, 50, 516517.

[22] Joshi, A. and Kostakis, G.C. (2004) An investigation of post-operative morbidity following iliac crest graft harvesting. British Dental Journal, 196, 167-171. doi:10.1038/sj.bdj.4810945 